\title{
Conducting crack propagation driven by electric fields in ferroelectric ceramics
}

\author{
Amir Abdollahi, Irene Arias \\ Laboratori de Càlcul Numèric (LaCàN), Departament de Matemàtica Aplicada III, \\ Universitat Politècnica de Catalunya (UPC), Campus Nord UPC-C2, E-08034 \\ Barcelona, Spain
}

\begin{abstract}
Ferroelectric ceramics are susceptible to fracture under high electric fields, which are commonly generated in the vicinity of electrodes or conducting layers. In the present work, we extend a phase-field model of fracture in ferroelectric single crystals for the simulation of the propagation of conducting cracks under purely electrical loading. This is done by introducing the electrical enthalpy of a diffuse conducting layer into the phase-field formulation. Simulation results show an oblique crack propagation and crack branching from a conducting notch, forming a tree-like crack pattern in a ferroelectric sample under positive and negative electric fields. Microstructure evolution indicates the formation of tail-to-tail and head-to-head $90^{\circ}$ domains, which results in charge accumulation around the crack. The charge accumulation, in turn, induces a high electric field and hence a high electrostatic energy further driving the conducting crack. Salient features of the results are compared with experiments.
\end{abstract}

Key words: Conducting cracks, Ferroelectricity, Fracture, Phase-field models, Finite element analysis 


\section{Introduction}

Over the past decades, ferroelectric ceramics have found many applications in smart structures and adaptive systems due to their unique electromechanical properties. The use of these materials as actuators demands a large actuation capability, often only attainable under high electric fields.

The architecture of actuators commonly involves internal electrodes or conducting layers, which can intensify the applied electric fields in their vicinity. The electric fields, in turn, can induce an incompatible strain field or a high electrostatic (Columbic) force, which may cause the brittle ferroelec-

tric ceramic to crack. Therefore, it is necessary to understand the fracture
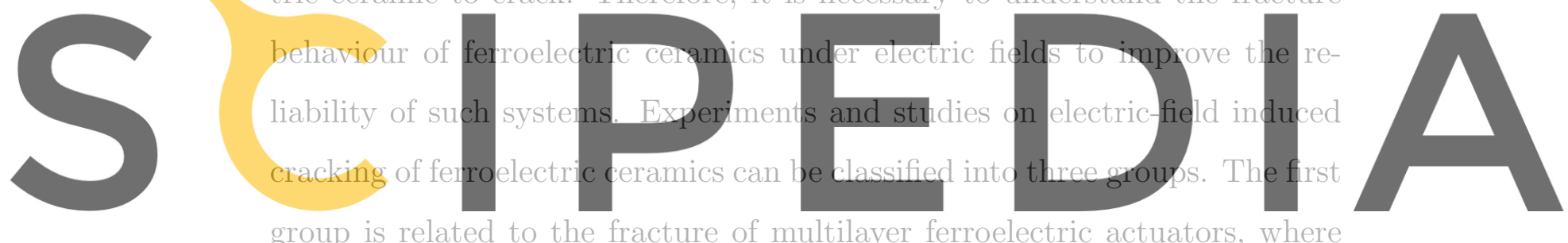

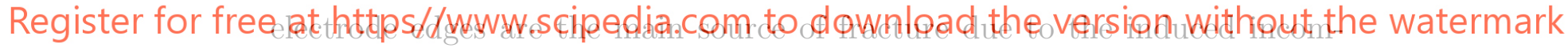

patible strain field $[1,2,3,4,5,6,7]$. Related theoretical models have been

proposed to understand the fracture of these actuators based on the theory of electrostrictive ceramics $[8,9,10,11,12]$, the linear theory of piezoelectricity $[13,14,15,16]$, and nonlinear approaches taking into account the ferroelectric and ferroelastic behaviors [17, 18, 4, 19, 7]. The second group of experiments have reported crack initiation and propagation from insulating notches, under electric fields applied perpendicularly to the notch. $[20,21,22,23,24,25,26,27,28,29,30]$. However, theoretical approaches show that electric fields, perpendicular to an insulating crack, decrease the total energy release rate, i.e. the electric fields prevent the crack propagation 
[31, 32, 33, 34, 35, 36, 37]. This discrepancy between the theoretical and experimental results has been discussed by Park and Sun [32]. They have concluded that the strain energy release rate is a proper fracture criterion for insulating cracks rather than the total energy release rate. Furthermore, there is another controversy on the different electrical boundary conditions on insulating crack faces since they affect the predicted energy release rates $[34,35,37]$. The third group of experiments have been performed on conducting cracks, where electric fields are applied parallel to the cracks, leading to the fracture of ferroelectric ceramics $[38,39,40,41,42,43]$. It is of technical relevance to investigate the electrical crack driving force on conducting
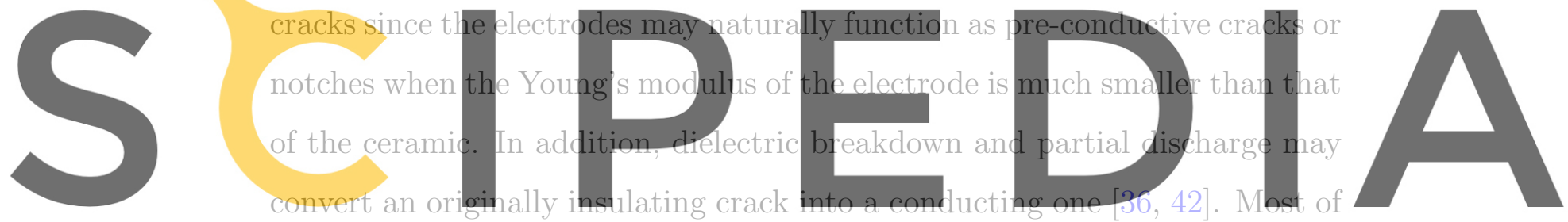

Register for free at hetps//www.scipedia.com to downior driving forcs to propagate the watermark conducting crack is the electrostatic force due to the accumulation of charges with the same sign at the crack tip. Theoretical models have also indicated that electric fields parallel to a conducting crack increase the total energy release rate $[31,44,9,45,36]$ and induce a large electrostatic driving force $[46,42]$. Therefore, in contrast to the insulating crack, both experiments and theoretical calculations consistently show an additional crack driving force produced by the electric field. The total energy release rate can also be considered as an appropriate fracture criterion for the conducting crack and the electrical boundary conditions on the crack faces are clear in comparison to those of an insulating crack [36]. For completeness, we mention that 
the fracture behavior of conducting cracks has been also investigated under combined mechanical and electrical loads [47, 48].

The above mentioned models for the conducting crack are useful to analyze the electromechanical fields near the crack tip. Nevertheless, most of these models are based on simplified electrostrictive or linear piezoelectricity theories, which do not consider the nonlinear effects of the domain switching in ferroelectrics. Related approaches have been developed to account for these effects and to investigate the toughening of conducting cracks due to domain switching $[49,50,51]$, relying on a simple small-scale switching criterion [52]. However, all of these models assume fixed crack configurations and they are
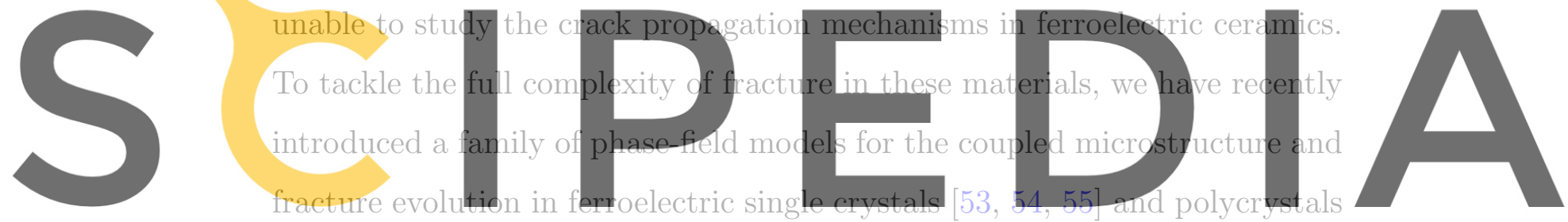

Register for free at https//wulations results show the potential af these phase-field models

to elucidate the fracture behavior of ferroelectric ceramics, observed in exper-

iments and applications. In particular, we have shown: (1) the slow-fast [53] and anisotropic crack propagation in ferroelectric single crystals [54], (2) the intergranular and transgranular modes of fracture in ferroelectric polycrystals [56], and (3) crack initiation patterns at electrode edges in multilayer actuators [57]. In all of these works we have considered insulating cracks under different electromechanical loading conditions. In this paper, we extend the phase-field theory to conducting cracks and investigating the crack propagation mechanisms under purely electrical loading.

The structure of the paper is as follows. In Section 2, we present a sum- 
mary of the phase-field model for the fracture of ferroelectric single crystals, introduced in Refs. [53, 54, 55]. Then, based on this model, we propose a phase-field formulation for conducting cracks. Numerical simulations are presented in Section 3, along with a discussion of the observed crack propagation patterns and fracture mechanisms. The last section is the conclusion of the paper.

2. Theory

\subsection{Phase-field model of fracture in ferroelectric single crystals}

The total electromechanical enthalpy of a possibly fractured ferroelec-
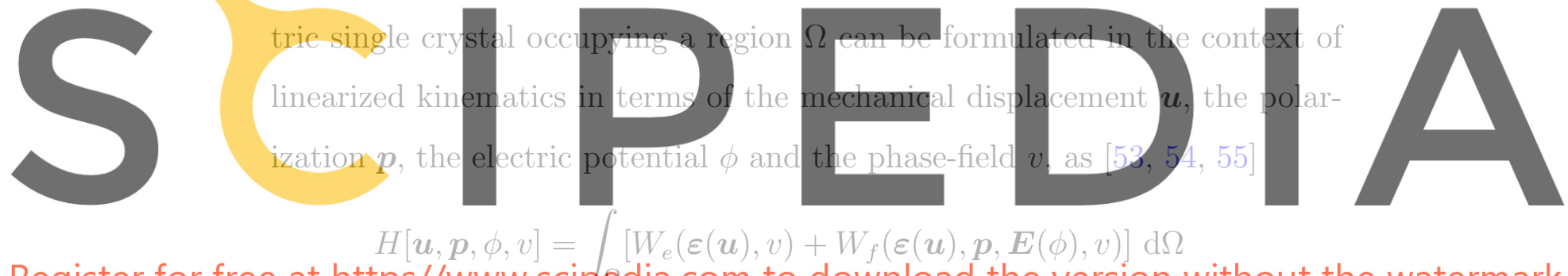

Register for free at https//www.scipedia.com to download the version without the watermark

$$
+G_{c} \int_{\Omega}\left[\frac{(1-v)^{2}}{4 \kappa}+\kappa|\nabla v|^{2}\right] \mathrm{d} \Omega
$$

where body loads, volume charges, tractions and surface charges have been ignored for simplicity. The first integral is referred to as total bulk energy of the material, where $W_{e}$ is the part of the bulk energy density associated with the strain $\varepsilon$ and $W_{f}$ is the electromechanical energy density associated with the ferroelectric response. The second integral takes the role of the surface energy, where $G_{c}$ is the critical energy release rate or the surface energy density in Griffith's theory [58]. The scalar field $v$ is the phase-field parameter describing a smooth transition in space between unbroken $(v=1)$ and broken $(v=0)$ states of the material. $\kappa$ is a positive regularization 
constant which regulates the size of the smeared fracture zone. The energy density $W_{e}$ is written as

$$
W_{e}(\varepsilon, v)=\kappa_{0} \frac{\operatorname{tr}^{-}(\varepsilon)^{2}}{2}+\left(v^{2}+\eta_{\kappa}\right)\left(\kappa_{0} \frac{\operatorname{tr}^{+}(\varepsilon)^{2}}{2}+\mu \varepsilon_{D} \cdot \varepsilon_{D}\right)
$$

where $\kappa_{0}$ and $\mu$ are the bulk and shear modulus of the material, respectively.

The trace of the strain tensor $\varepsilon$ is decomposed in positive and negative parts as $\operatorname{tr}^{+}=\max (\operatorname{tr}(\varepsilon), 0)$ and $\operatorname{tr}^{-}=\max (-\operatorname{tr}(\varepsilon), 0)$ and $\varepsilon_{D}$ are the deviatoric components of the strain tensor. This decomposition is introduced to distinguish the contributions to the strain energy due to compression, expansion,
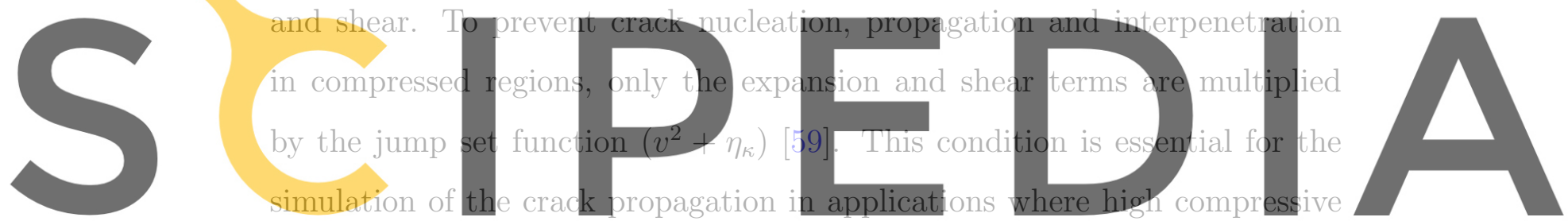

stresses are induced [54]. The parameter $\eta_{\kappa}$ is a small (relative to $\kappa$ ) residual Register for free at https//wWW.scipedia.com to download the version without the watermark stiffness to avoid the singularity of the bulk energy in fully fractured regions

of the domain.

The electromechanical energy density $W_{f}$ associated with polarization $\boldsymbol{p}$, electric potential $\phi, \varepsilon$ and $v$ is given as

$$
W_{f}(\boldsymbol{\varepsilon}, \boldsymbol{p}, \phi, v)=\left(v^{2}+\eta_{\kappa}\right)[U(\nabla \boldsymbol{p})+W(\boldsymbol{p}, \boldsymbol{\varepsilon})]+\chi(\boldsymbol{p})-\boldsymbol{E} \cdot \boldsymbol{p}-\frac{\varepsilon_{0}}{2}|\boldsymbol{E}|^{2},
$$

where $\boldsymbol{E}$ is the electric field defined as $\boldsymbol{E}=-\nabla \phi, U$ is the domain wall energy density penalizing sharp variations in the polarization, $\chi$ is the phase separation potential, $W$ is the electromechanical coupling energy density and $\varepsilon_{0}$ is the vacuum permittivity. The energy densities $\chi$ and $W$ penalize 
deviations from the spontaneous polarizations and strains of the material, hence introducing the anisotropy and nonlinearity of ferroelectric materials. The combination of the energy functions $\chi, W$ and $W_{e}$ is the total LandauDevonshire energy density furnishing a multi-well energy landscape with four minima in two dimensions corresponding to the four variants of the tetragonal phase. The detailed formulation of these energy functions and the related material constants are presented in A.

In addition to the asymmetric fracture response in tension and compression, this particular formulation of the phase-field model encodes the following assumed crack conditions: (1) traction-free, (2) electrically permeable,
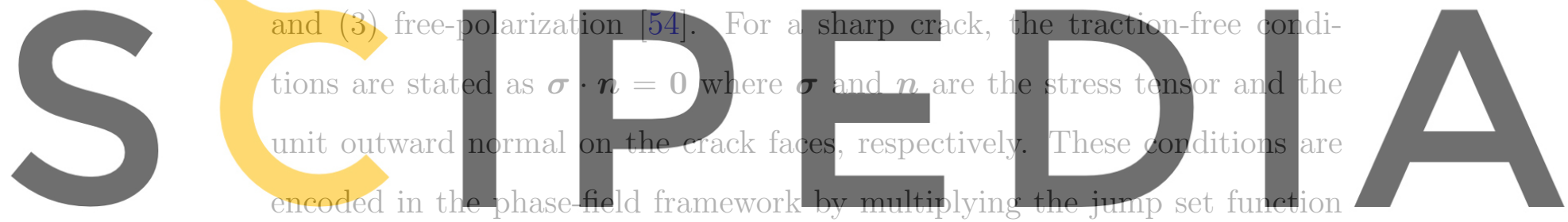

Register for free at hetes $v^{2} /$ WwW.

a consequence, the energy terms associated with the strains $\varepsilon$ vanish inside

the fracture zone $(v=0)$ and outside the compressed regions. The permeable conditions assume that crack faces are closed and the electrical fields are not perturbed by the presence of the crack. These conditions have been checked to be realistic for non-conducting cracks based on the observed fracture behavior of ferroelectric ceramics $[60,61,62]$. To encode the permeable conditions, the last two terms in Eq. (3), associated with the electric field $\boldsymbol{E}$, are not multiplied by the the jump set function.

In contrast to piezoelectrics, the modeling of cracks in ferroelectric materials requires imposing boundary conditions on the polarization on the crack 
faces. Free-polarization boundary conditions are commonly assumed, implying that the polarization distribution near each side of the crack is unaffected by it, and hence dictated by the bulk material model [63, 64]. Mathematically, they are written as

$$
\nabla \mathbf{p}^{+} \cdot \boldsymbol{n}=\nabla \mathbf{p}^{-} \cdot \boldsymbol{n}=\mathbf{0}
$$

where the superscripts + and - denote the top and bottom crack faces. We introduce these conditions in the phase-field framework by suppressing the gradient of polarization inside the fracture zone, i.e. multiplying $U$ by the jump set function $\left(v^{2}+\eta_{\kappa}\right)$ in Eq. (3).
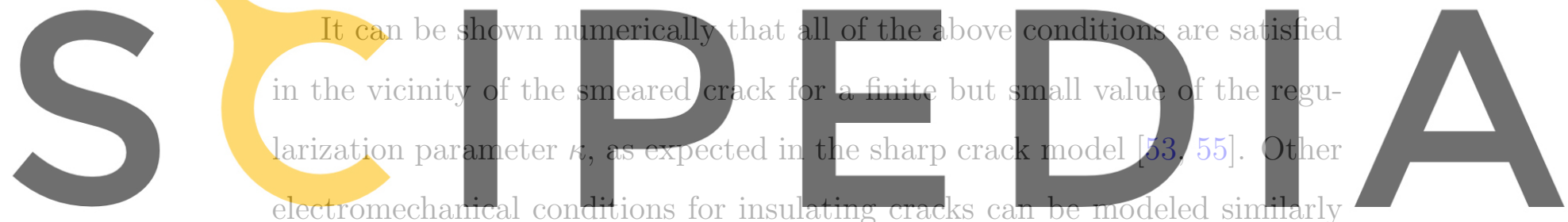

Register for free 5 at·https//www.scipedia.com to download the version without the watermark

To capture interactions between the fracture and the microstructure processes, the crack propagation should not be overwhelmingly faster than the microstructure evolution, and vice versa. In practice, the relative kinetics of the microstructure evolution and the crack propagation gives the two phenomena a chance to interact. In the absence of detailed experimental or fundamental information on these kinetics, $v$ is selected together with the polarization as primary order parameters and finite mobilities are introduced for the fracture and microstructure processes. Then, the time evolution of the system results from the gradient flows of the primary order parameters, assuming that the displacement and the electric field adjust immediately to 
mechanical and electrostatic equilibrium (with infinite mobility), i.e.

$$
\begin{aligned}
\alpha \int_{\Omega} \dot{p}_{i} \delta p_{i} \mathrm{~d} \Omega & =-\delta H[\boldsymbol{u}, \boldsymbol{p}, v, \phi ; \delta \boldsymbol{p}], \\
\beta \int_{\Omega} \dot{v} \delta v \mathrm{~d} \Omega & =-\delta H[\boldsymbol{u}, \boldsymbol{p}, v, \phi ; \delta v], \\
0 & =\delta H[\boldsymbol{u}, \boldsymbol{p}, v, \phi ; \delta \boldsymbol{u}], \\
0 & =-\delta H[\boldsymbol{u}, \boldsymbol{p}, v, \phi ; \delta \phi]
\end{aligned}
$$

where $1 / \alpha>0$ and $1 / \beta>0$ are the mobilities of the processes. The form of the variations of the electromechanical enthalpy is given in B, along with a simple algorithm to solve the coupled system.

Two constraints are also considered for solving the equation of the frac-
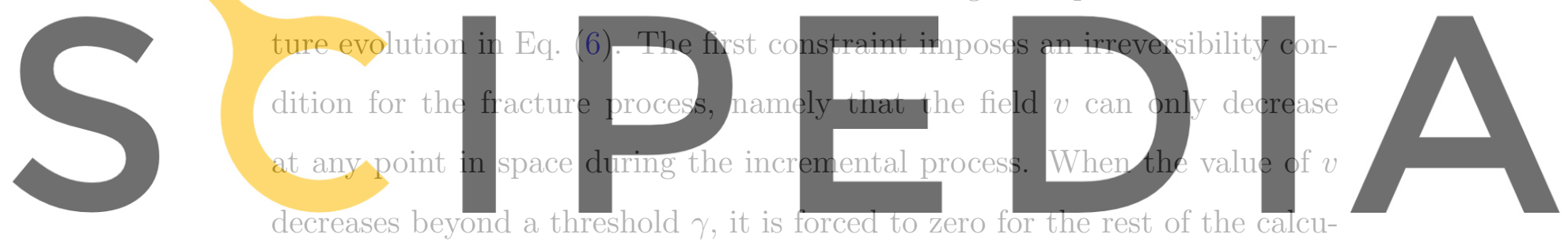

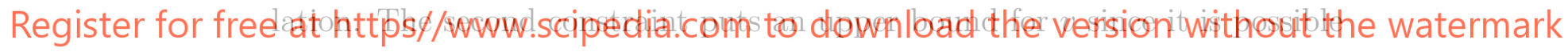

in electromechanical fracture that in some regions, a non-positive crack driv-

ing force is induced by the load. It is favorable then to localize in these regions high values of $v$, much above one. Such anomalous localization zones are not physically meaningful. This issue manifests itself in sharp models of electromechanical crack propagation, with the negative energy release rates found at high applied electric fields [37]. Numerically, we deal with this issue by limiting the maximum value of $v$ to one.

\subsection{Phase-field model for conducting cracks}

A conducting crack is modeled by assuming that the crack faces are coated with perfectly conducting electrodes. Therefore, the electric potential is con- 
stant along the crack and the electric field inside the crack gap is zero. Experimentally, these conditions can be implemented by filling the crack gap with a conducting fluid or electrolyte such as $\mathrm{NaCl}$ solution $[47,48]$ or silver paint $[39,41]$. The crack-gap filling electrolyte acts as an internal conducting layer with very large permittivity. In the context of phase-field models, this layer can be defined in a smeared way by modifying the electromechanical enthalpy in Eq. (3) in such a way that the permittivity reaches infinity inside the fractured zone $(v=0)$. This is done by multiplying the vacuum permittivity $\varepsilon_{0}$ by the jump set function $1 /\left(v^{2}+\varepsilon_{c}^{-1}\right)$, where $\varepsilon_{c}$ is the relative permittivity of the fractured zone. Then the electromechanical enthalpy
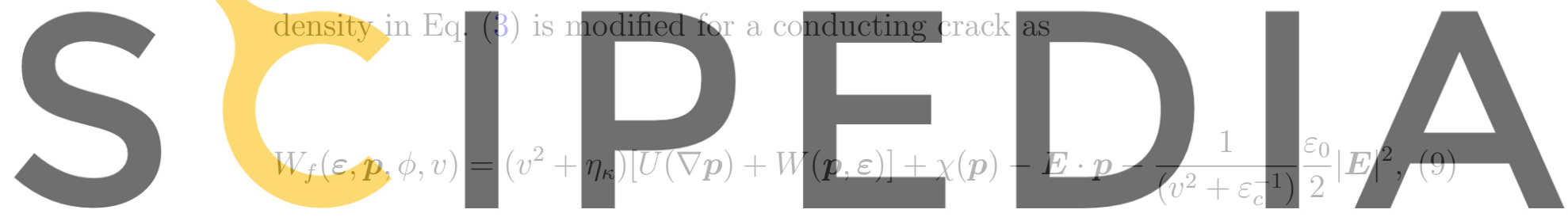

If $\varepsilon_{c}$ is chosen sufficiently large, the last term in Eq. (9) will represent the elecRegister for free at https//www.scipedia.com to download the version without the watermark

trical enthalpy of a difluse conducting layer, as shown schematically in Fig. 1.

The jump set function $1 /\left(v^{2}+\varepsilon_{c}^{-1}\right)$ does not alter the vacuum permittivity outside of the fracture zone $(v=1), \varepsilon_{c}^{-1}$ being an infinitesimal value. On the other hand, this function attains its maximum for $v=0$, thereby encoding the conditions of a conducting crack, with a large relative permittivity $\varepsilon_{c}$ inside the fractured zone. A partially conducting layer appears as a smooth transition between the insulating vacuum and conducting layers $(0<v<1)$. When the regularization parameter $\kappa$ tends to zero, this transition becomes sharper and the semi-conducting layer tends to disappear. We show in Fig. 8 that indeed this method produces numerical solutions, satisfying the desired conditions of a sharp conducting crack in an accurate way for a small value 
of the regularization parameter.

Note that this formulation together with the energy density $W_{e}$ in Eq. (2) account for the asymmetric behavior in tension and compression and encode also the free-polarization conditions on the conducting crack, as discussed in Section 2.1. As sustained by several investigations [47, 48], we consider the critical energy release rate $G_{c}$ as a valid fracture criterion for conducting cracks. Therefore, the total electromechanical enthalpy in Eq. (1), with $W_{f}$ given in Eq. (9), is also valid for conducting cracks.

\section{Numerical simulation}

\subsection{Computational domain and parameter setting}

To perform numerical simulations, we consider a square domain presented in Fig. 2 of size $L_{1}=10 \mathrm{~mm}$. The material constants are chosen to fit the behavior of single crystals of barium titanate $\left(\mathrm{BaTiO}_{3}\right)$ at room temperature. The initial polarization $\boldsymbol{p}_{0}$ is along the positive horizontal direction with a magnitude of $0.26 \mathrm{Cm}^{-2}$. A deep pre-notch is introduced in the model, parallel to the initial polarization, to facilitate the crack initiation, while avoiding the boundary effects on the notch tip. The notch dimensions are chosen as $L_{n}=5 \mathrm{~mm}$ and $W=50 \mathrm{~nm}$. To create a conducting notch, the electric potential is fixed to zero on the notch surface. The electric potential is also set to 0 and $V$ on the left and right sides of the domain, respectively. Therefore, different electric fields can be applied in the horizontal direction by giving different values to the driving voltage $V$. The model is discretized with ap-

proximately 100,000 triangular finite elements of different sizes. A fine mesh 
with element size $h=2 \mathrm{~nm}$ is generated in a small square region of interest of size $L_{2}=200 \mathrm{~nm}$ around the notch, presented in Fig. 2(b), where fracture is expected. This element size is small enough relative to the ferroelectric domain walls with a thickness of few nanometers $[65,66]$. The rest of the domain is meshed with larger elements. Note that in the phase-field model, a domain wall scaling parameter $a_{0}$ regulates the size of the domain walls. This parameter has to be chosen such that the variation of the polarization can be resolved by the discretization, particularly across the coarse elements away from the notch tip, while the domain walls near the tip remain sufficiently sharp in the order of their physical thickness. The adjustment of the parameter $a_{0}$ to the element size is essential to avoid domain wall pinning at the larger elements and does not affect the results inside the region of interest. See A for further details on setting this parameter.

An electric field $E=-V / L_{1}$ of up to $1.4 \mathrm{KV} / \mathrm{mm}$ is applied incrementally in the positive and negative directions by increasing the driving voltage $V$ in 75, 000 quasi-static load increments of $\Delta V= \pm 18.67 \times 10^{-2} \mathrm{~V}$. A pseudotime step $\Delta t=10^{-2}$ also leads to convergent and accurate solutions for the explicit integration of the gradient flow equations in Eqs. (5) and (6). A relative permittivity $\varepsilon_{c}=10^{8}$ has been found large enough to accurately reproduce the conditions of a conducting crack.

The simulations are performed following a simple algorithm presented in B. The remaining constants are chosen as follows. The regularization parameter $\kappa$ is set to twice the finest element size as $\kappa=4$ nm, i.e. $h / \kappa=0.5$, the threshold to detect the irreversibly fractured regions to $\gamma=2 \times 10^{-2}$, the residual stiffness to $\eta_{\kappa}=10^{-4}$, the tolerances to achieve steady states for 
ferroelectric domains and fracture processes to $\delta_{\text {ferro }}=\delta_{v \text { field }}=10^{-3}$, and the inverse mobilities to $\alpha=10$ and $\beta=45$. The simulations are carried out on parallel processors using the finite element library of the Kratos multiphysics package [67].

\subsection{Numerical results and discussion}

Figure 3 presents three snapshots of the crack propagation in the small neighborhood of the notch tip under positive applied electric fields. As the electric field increases, the $v$ field starts to decrease at two points of the circular tip until it reaches the threshold to be considered permanently fractured $(v<0.02)$, and the cracks initiate, see Fig. 3(a). By further increasing the load, the field $v$ evolves along two directions from the notch tip, forming a tree-like crack pattern. A similar crack initiation, oblique crack propagation and branching is also observed under negative applied electric fields, as shown in Fig. 4. The simulations are stopped at load $|E|=1.4 \mathrm{KV} / \mathrm{mm}$ because by further increasing the load, the cracks propagate though the coarse mesh zone, where they cannot be resolved numerically. Longer cracks can be obtained by employing an adaptive mesh refinement strategy, which is beyond the scope of the present paper.

The main difference between the results of the positive and negative electric fields is that the cracks under the positive field are more curved and tend to branch again as the load increases. Interestingly, an oblique crack propagation is also observed in experiments of conducting cracks in ferroelectric ceramics under purely electrical loading [41], where a rough fracture surface was observed. The crack patterns in our simulations can be conceived as the 
initial stages of the experimentally observed tree-like cracks [20]. Other experiments have also reported the formation of a micro-cracks network driven by electric fields [48]. Furthermore, our simulation results show that the cracks are longer under the negative electric field, cf. Fig. 3 and 4. Again, this is in agreement with experiments showing that electric fields in opposite to the initial polarization strongly facilitate the conducting fracture [48]. Crack closure is also observed in the simulation under the positive electric field. In this situation, the crack faces cease to be traction-free since the proposed model prevents crack interpenetration in compressed regions, see Eq. (2). This is an important aspect of electromechanical crack propagation addressed by several studies on conducting cracks in ferroelectrics $[47,68,48]$. These studies have consistently indicated that crack closure occurs for a conducting crack under an electric field applied in the poling direction, which is in agreement with our results.

The origin of the observed crack patterns can be found in the domain switching during crack growth. Figures 5 and 6 present snapshots of the domain evolution under the positive and negative electric fields, respectively. Charge accumulation occurs around the cracks through the formation of tailto-tail and head-to-head $90^{\circ}$ domains in Fig. 5 and 6, respectively. This charge accumulation with the same sign induces a high electric field at the crack tip, which in turn leads to a high electrostatic energy (last term in Eq. (9)) for driving the crack. The polarization components along a crosssection of the crack under the negative electric field are presented in Fig. 7. Due to the free-polarization conditions (see Eq. (4)), the polarization components approach with zero slope the edges of the smeared crack and 
they follow a smooth transition across the crack. This smooth transition becomes sharper as the value of the regularization parameter $\kappa$ decreases towards zero. The same sign of the polarization components on the two sides of the crack clearly indicates the head-to-head $90^{\circ}$ domains and hence the charge accumulation. For the sake of brevity, a similar figure for the positive electric field (with the opposite sign of the polarization components) is not shown.

Figure 8 presents the electric field magnitude during the load steps obtained at a point in the path of the conducting crack under the negative electric field. The electric field intensification is obvious when the crack reaches this point at load step a. On the other hand, the electric field suddenly vanishes when the crack passes through this point at load step b, i.e. the electric potential becomes constant in the fractured zone as expected. This provides numerical evidence that the conditions of a conducting crack are accurately satisfied by the proposed phase-field model. Since the cracks are conducting and connected to the pre-notch, the electric potential inside the fractured zone is the same as that inside the pre-notch, which is assumed to be zero. We note that the same simulation results are obtained by fixing the electric potential to a non-zero value on the pre-notch. In this case, the driving voltage $V$ should be increased by the non-zero value to produce the same electric field. The nucleation of new twins also induces a high electric field, which is responsible for the initiation of small crack branches under the positive electric field, see Fig. 3(c) and 5(c). Polarization reversal in front of the notch is obvious in Fig. 6 under the negative electric field. 


\section{Conclusions}

We perform, to the best of our knowledge, the first simulation of conducting crack propagation in ferroelectric ceramics under purely electrical loading. Our simulations agree qualitatively with the experimental record and provide new insight on this complex process. For this purpose, we extend to conducting cracks, a phase-field model of fracture in ferroelectric ceramics by introducing the electrical enthalpy of a diffuse conducting layer into the phase-field formulation. Simulation results show an oblique crack propagation and crack branching from a conducting notch, forming a treelike crack pattern in a ferroelectric sample. The origin of the observed crack patterns have been found in the formation of tail-to-tail and head-to-head $90^{\circ}$ domains which results in a charge accumulation around the crack. The charge accumulation, in turn, induces a high electrostatic energy for driving the conducting cracks. A negative electric field induces a larger driving force with respect to the positive one. All these observations agree with experiments.

We also suggest that dielectric breakdown mechanisms can be modeled in a similar way to that of conducting crack propagation since dielectric breakdown occurs via the formation of conducting tubular channels under high electric fields [44]. Dielectric breakdown has been also observed during the propagation of conducting cracks [39, 41, 43, 69]. The formation and propagation of the tubular channels can shield or reduce the electric field, resulting in a decrease in the driving force of conducting cracks, and they can also affect the microstructure of the material. Furthermore, the propagation of conducting cracks under combined electro-mechanical loading can 
also be studied with the proposed model. These topics are currently under investigation.

\section{Acknowledgements}

The authors gratefully acknowledge the support of the Ministerio de Ciencia e Innovación (DPI2011-26589).

\section{A. Energy functions and material parameters}

The energy functions $U, W$ and $\chi$ in Eq. (3) are chosen following [70, 71], adapted to a plane polarization and plane strain state:

$$
\begin{gathered}
U\left(p_{i, j}\right)=\frac{a_{0}}{2}\left(p_{1,1}^{2}+p_{1,2}^{2}+p_{2,1}^{2}+p_{2,2}^{2}\right), \\
W\left(p_{i}, \varepsilon_{j k}\right)=-\frac{b_{1}}{2}\left(\varepsilon_{11} p_{1}^{2}+\varepsilon_{22} p_{2}^{2}\right)-\frac{b_{2}}{2}\left(\varepsilon_{11} p_{2}^{2}+\varepsilon_{22} p_{1}^{2}\right) \\
-b_{3}\left(\varepsilon_{21}+\varepsilon_{12}\right) p_{1} p_{2},
\end{gathered}
$$

$$
\begin{aligned}
\chi\left(p_{i}\right) & =\alpha_{1}\left(p_{1}^{2}+p_{2}^{2}\right)+\alpha_{11}\left(p_{1}^{4}+p_{2}^{4}\right)+\alpha_{12}\left(p_{1}^{2} p_{2}^{2}\right)+\alpha_{111}\left(p_{1}^{6}+p_{2}^{6}\right)+\alpha_{112}\left(p_{1}^{2} p_{2}^{4}+p_{2}^{2} p_{1}^{4}\right) \\
& +\alpha_{1111}\left(p_{1}^{8}+p_{2}^{8}\right)+\alpha_{1112}\left(p_{1}^{6} p_{2}^{2}+p_{2}^{6} p_{1}^{2}\right)+\alpha_{1122}\left(p_{1}^{4} p_{2}^{4}\right)
\end{aligned}
$$

where $a_{0}$ is the scaling parameter of the domain wall energy, $b_{i}(i=1,2,3)$ are the constants of the coupling terms between strain and polarization and $c_{i}(i=1,2,3)$ are the elastic constants. The phase separation energy $\chi$ is improved by adding the eighth-order terms with coefficients $\alpha_{1111}, \alpha_{1112}$ and 
$\alpha_{1122}$ to reproduce the dielectric behavior of barium titanate $\left(\mathrm{BaTiO}_{3}\right)$ single crystals $[72,73]$. The eighth-order term with coefficient $\alpha_{1122}$ enables the model to fit the dielectric constants while retaining a reasonable energy barrier for $90^{\circ}$ domain switching in the tetragonal phase $[74,75] . \alpha_{1}$ is linearly dependent on temperature and its negative value makes the cubic phase unstable. $\alpha_{111}$ is estimated by fitting the spontaneous polarization of the tetragonal phase. $\alpha_{112}$ and $\alpha_{1112}$ are fitted to the dielectric permittivity perpendicular to the spontaneous polarization. $\alpha_{11}, \alpha_{12}$ and $\alpha_{1111}$ are evaluated from linear and nonlinear dielectric measurements above the Curie temperature [73]. The constants are chosen to fit the behavior of single crystals of barium titanate $\left(\mathrm{BaTiO}_{3}\right)$ at room temperature with a spontaneous polarization $p_{0}=0.26 \mathrm{Cm}^{-2}$, relative spontaneous strains $\varepsilon_{a}=-0.44 \%$ along a-axis and $\varepsilon_{c}=0.65 \%$ along c-axis $[74,73]$. The domain wall scaling parameter is set as $a_{0}=37 h \mathrm{Vm}^{2} \mathrm{C}^{-1}$, where $h$ is the element size. The $90^{\circ}$ domain walls width is then spanned with 3-5 elements of size $h=2 \mathrm{~nm}$ near the notch tip in the simulations, corresponding to 6-10 nanometers, in the order of experimentally measured values in tetragonal ferroelectric ceramics $[65,66]$. Furthermore, increasing $a_{0}$ with the element size results in a smooth variation of the polarization across coarse elements away from the notch tip.

Normalized parameters are presented in table 1 through the following normalizations: $x_{i}^{\prime}=x_{i} \sqrt{c_{0} / l_{0}} / p_{0}, \mu^{\prime}=\mu / c_{0}, \kappa_{0}^{\prime}=\kappa_{0} / c_{0}, p_{i}^{\prime}=p_{i} / p_{0}, \varepsilon_{0}^{\prime}=$ $\varepsilon_{0} c_{0} / p_{0}^{2}, \phi^{\prime}=\phi / \sqrt{l_{0} c_{0}}, \alpha_{1}^{\prime}=\alpha_{1} p_{0}^{2} / c_{0}, \alpha_{11}^{\prime}=\alpha_{11} p_{0}^{4} / c_{0}, \alpha_{12}^{\prime}=\alpha_{12} p_{0}^{4} / c_{0}, \alpha_{111}^{\prime}=$ $\alpha_{111} p_{0}^{6} / c_{0}, \alpha_{112}^{\prime}=\alpha_{112} p_{0}^{6} / c_{0}, \alpha_{1111}^{\prime}=\alpha_{1111} p_{0}^{8} / c_{0}, \alpha_{1112}^{\prime}=\alpha_{1112} p_{0}^{8} / c_{0}, \alpha_{1122}^{\prime}=$ $\alpha_{1122} p_{0}^{8} / c_{0}, b_{i}^{\prime}=b_{i} p_{0}^{2} / c_{0}$ and $c_{i}^{\prime}=c_{i} / c_{0}$, where $i=1,2,3, c_{0}=1 \mathrm{GPa}$ and $l_{0}=3.7 \times 10^{-9} \mathrm{Vm}^{3} \mathrm{C}^{-1}$. Considering Young's modulus, Poisson's ratio and 
fracture toughness for $\mathrm{BaTiO}_{3}$ as $E=100 \mathrm{GPa}, \nu=0.37$, and $K_{c}=0.49$ $\mathrm{MPa} \sqrt{m}$, respectively, the value of the critical energy release rate in plane strain is obtained as $G_{c}=\left(1-\nu^{2}\right) K_{c}^{2} / E=2 \mathrm{Jm}^{-2}$. The value of normalized critical energy release rate is then calculated as $G_{c}^{\prime}=G_{c} \sqrt{1 / l_{0} c_{0}} / p_{0}=4$.

\section{B. Weak form of the governing equations and solution algorithm}

The weak forms of the gradient flows in Eqs. (5) and (6), together with the equations for mechanical and electrostatic equilibria in Eqs. (7) and (8), follow from

$$
\begin{aligned}
\alpha \int_{\Omega} \dot{p}_{i} \delta p_{i} \mathrm{~d} \Omega & =-\delta H[\boldsymbol{u}, \boldsymbol{p}, v, \phi ; \delta \boldsymbol{p}] \\
& =-\int_{\Omega}\left[\left(v^{2}+\eta\right)\left(\frac{\partial U}{\partial p_{i, j}} \delta p_{i, j}+\frac{\partial W}{\partial p_{i}} \delta p_{i}\right)+\frac{\partial \chi}{\partial p_{i}} \delta p_{i}+\phi_{, i} \delta p_{i}\right] \mathrm{d} \Omega \\
\beta \int_{\Omega} \dot{v} \delta v \mathrm{~d} \Omega= & -\delta H[\boldsymbol{u}, \boldsymbol{p}, v, \phi ; \delta v] \\
= & -\int_{\Omega} \frac{\partial\left(W_{e}+W_{f}\right)}{\partial v} \delta v \mathrm{~d} \Omega-2 G_{c} \int_{\Omega}\left(\frac{v-1}{4 \kappa} \delta v+\kappa v_{, i} \delta v_{, i}\right) \mathrm{d} \Omega \\
0 & \delta H[\boldsymbol{u}, \boldsymbol{p}, v, \phi ; \delta \boldsymbol{u}]=\int_{\Omega} \frac{\partial\left(W_{e}+W_{f}\right)}{\partial \varepsilon_{i j}} \delta \varepsilon_{i j} \mathrm{~d} \Omega \\
& 0 \quad-\delta H[\boldsymbol{u}, \boldsymbol{p}, v, \phi ; \delta \phi]=\int_{\Omega} \frac{\partial W_{f}}{\partial E_{i}} \delta E_{i} \mathrm{~d} \Omega
\end{aligned}
$$

where the elastic strain $\varepsilon_{i j}$ and electric field $E_{i}$ are associated with the mechanical displacement $u_{i}$ and the electric potential $\phi$, respectively, as $\varepsilon_{i j}=1 / 2\left(u_{i, j}+u_{j, i}\right)$ and $E_{i}=-\phi_{, i}$. Equations (13) and (14) are discretized 
in time with an explicit scheme from time $t_{m}$ to $t_{m+1}=t_{m}+\Delta t$. Note that the minimization in Eq. (15) is non-smooth, and a quasi-Newton algorithm is applied to solve the mechanical equilibrium problem following [59].

A simple algorithm to solve the coupled system in a straightforward staggered approach is presented in Algorithm 1. This algorithm describes how to advance in one load step (or pseudo-time increment), and it is meant to achieve steady states for both ferroelectric domains and fracture processes in each load step. The functions $\boldsymbol{g}(w)$ and $f(w)$ encode the Dirichlet data for the mechanical displacement and electric potential as a function of the load step $w$. After reaching a steady state for both the polarization and the phase-field $v$, the values for $v^{n}, \boldsymbol{u}^{n}, \mathbf{p}^{n}$ and $\phi^{n}$ are recorded and the algorithm proceeds to the next load step $n+1$.

\section{References}

\section{References}

[1] Uchino K, Furuta A. ISAF 92 : Proc 8th IEEE Int Symp Appl Ferroelectrics $1992 ;: 195$.

[2] Furuta A, Uchino K. J Amer Ceram Soc 1993;76:1615.

[3] Aburatani H, Harada S, Uchino K, Furuta A, Fuda Y. JPN J Appl Phys 1 1994;33:3091.

[4] Lucato SLDE, Lupascu DC, Kamlah M, Rodel J, Lynch CS. Acta Mater 2001;49:2751.

[5] Koh JH, Jeong SJ, Ha MS, Song JS. Ceram Int 2004;30:1863. 
[6] Liu TQ, Oates WS, Wan S, Lynch CS. J Intell Mater Syst Struct $2005 ; 16: 373$.

[7] Zhao XJ, Liu B, Fang DN. Int J Plasticity 2010;26:533.

[8] Winzer SR, Shankar N, Ritter AP. J Amer Ceram Soc 1989;72:2246.

[9] Yang W, Suo Z. J Mech Phys Solids 1994;42:649.

[10] Hao TH, Gong X, Suo Z. J Mech Phys Solids 1996;44:23.

[11] Gong X, Suo Z. J Mech Phys Solids 1996;44:751.

[12] Ru CQ, Mao X, Epstein M. J Mech Phys Solids 1998;46:1301.

[13] Ru CQ. J Mech Phys Solids 2000;48:693.

[14] Ye RQ, He LH. Int J Solids Struct 2001;38:6941.

[15] Li XF, Tang GJ. Mech Research Commun 2003;30:345.

[16] Guo XH, Fang DN, Soh AK, Kim HC, Lee JJ. Acta Mech Sinica $2006 ; 22: 34$.

[17] Kamlah M. Cont Mech Therm 2001;13:219.

[18] Kamlah M, Bohle U. Int J Solids Struct 2001;38:605.

[19] Elhadrouz M, Zineb TB, Patoor E. Int J Eng Sci 2006;44:996.

[20] Lynch C, Yang W, Collier L, Suo Z, McMeeking RM. Ferroelectrics 1995;166(1-4):11. 
[21] Lynch CS, Chen L, Suo Z, Mcmeeking RM, Yang W. J Intell Mater Syst Struct 1995;6:191.

[22] Lucato SLDE, Bahr HA, Pham VB, Lupascu DC, Balke H, Rodel J, et al. J Mech Phys Solids 2002;50:2333.

[23] Liu B, Fang D, Hwang KC. Mater Lett 2002;54(5-6):442.

[24] Lucato SLDE, Bahr HA, Pham VB, Lupascu DC, Balke H, Rodel J, et al. J Eur Ceram Soc 2003;23:1147.

[25] Niefanger R, Pham VB, Schneider GA, Bahr HA, Balke H, Bahr U. Acta Mater 2004;52(1):117.

[26] Shieh J, Huber JE, Fleck NA. J Eur Ceram Soc 2006;26:95.

[27] Westram I, Lupascu D, Roedel J, Laskewitz B, Kamlah M. J Amer Ceram Soc 2007;90:2849.

[28] Westrain I, Oates WS, Lupascu DC, Roedel J, Lynch CS. Acta Mater 2007;55:301.

[29] Jiang Y, Fang D. Mater Lett 2007;61:5047.

[30] Jiang Y, Zhang Y, Liu B, Fang D. Acta Mater 2009;57:1630.

[31] Suo Z. Amer Soc Mech Eng 1991;24:1.

[32] Park SB, Sun CT. J Am Ceram Soc 1995;78(6):1475.

[33] McMeeking RM. Eng Fract Mech 1999;64:217.

[34] McMeeking RM. Eng Fract Mech 2004;71:1149. 
[35] Landis CM. Int J Solids Struct 2004;41(22-23):6291.

[36] Schneider GA. Annu Rev Mater Res 2007;37:491.

[37] Li WY, McMeeking RM, Landis CM. Eur J Mech A-Solids $2008 ; 27(3): 285$.

[38] Fu R, Qian CF, Zhang TY. Appl Phys Lett 2000;76:126.

[39] Zhang TY, Wang TH, Zhao MH. Acta Mater 2003;51:4881.

[40] Zhang TY, Gao CF. Theor Appl Fract Mech 2004;41:339.

[41] Zhang TY, Liu GN, Wang Y. Acta Mater 2004;52:2025.

[42] Zhang TY, Liu G, Wang T, Tong P. Eng Fract Mech 2007;74:1160.

[43] Beom HG, Jeong KM, Park JY, Lin S, Kim GH. Eng Fract Mech 2009;76:2399.

[44] Suo Z. J Mech Phys Solids 1993;41:1155.

[45] Qian TY, Tong CF, Zhang P. Int J Solids Struct 1998;35(17):2121.

[46] Zhang TY, Zhao MH, Liu GN. Acta Mater 2004;52:2013.

[47] Heyer V, Schneider GA, Balke H, Drescher J, Bahr HA. Acta Mater 1998;46:6615.

[48] Gehrig F, Jelitto H, Schneider GA. Acta Mater 2008;56:222.

[49] Zeng RKND, Rajapakse X. Acta Mater 2001;49(5):877.

[50] Jeong KM, Beom HG. Smart Mater Struct 2004;13(2):275. 
[51] Beom HK, Youn SK. Int J Solids Struct 2004;41(1):145.

[52] Hwang SC, Lynch CS, McMeeking RM. Acta Mater 1995;43:2073.

[53] Abdollahi A, Arias I. Acta Mater 2011;59:4733.

[54] Abdollahi A, Arias I. Model Simul Mater Sci Eng 2011;19:074010.

[55] Abdollahi A, Arias I. J Mech Phys Solids 2012;60:2100.

[56] Abdollahi A, Arias I. Int J Fract 2012;174:3.

[57] Abdollahi A, Arias I. Smart Mater Struct 2012;21:094011.

[58] Griffith AA. Philos Trans Royal Soc London 1921;A221:163.

[59] Amor H, Marigo JJ, Maurini C. J Mech Phys Solids 2009;57:1209.

[60] Schneider GA, Felten F, McMeeking RM. Acta Mater 2003;51:2235.

[61] Haeusler C, Jelitto H, Neumeister P, Balke H, Schneider GA. Int J Fract 2009;160:43.

[62] Engert AR, Neumeister P, Mecklenburg M, Jelitto H, Balke H, Schneider GA. J Eur Ceram Soc 2011;31:531.

[63] Vendik OG, Zubko SP. J Appl Phys 2000;88:5343.

[64] Wang J, Kamlah M. Eng Fract Mech 2010;77:3658.

[65] Stemmer S, Streiffer SK, Ernst F, Ruhle M. Phil Mag A 1995;71:713.

[66] Floquet N, Valot CM, Mesnier MT, Niepce JC, Normand L, Thorel A, et al. J Phys III 1997;7:1105. 
[67] Dadvand P, Rossi R, Onate E. Arch Comput Methods Eng 2010;17:253.

[68] Ru CQ. Acta Mater 1999;47:4683.

[69] Lin S, Beom H, Tao D. Acta Mech 2010;210:47.

[70] Devonshire AF. Philos Mag 1949;40:1040.

[71] Devonshire AF. Philos Mag 1951;42:1065.

[72] Li YL, Cross LE, Chen LQ. J Appl Phys 2005;98:064101.

[73] Wang YL, Tagantsev AK, Damjanovic D, Setter N, Yarmarkin VK, Sokolov AI, et al. J Appl Phys 2007;101:104115.

[74] Zhang W, Bhattacharya K. Acta Mater 2005;53:185.

[75] Su Y, Landis CM. J Mech Phys Solids 2007;55:280. 


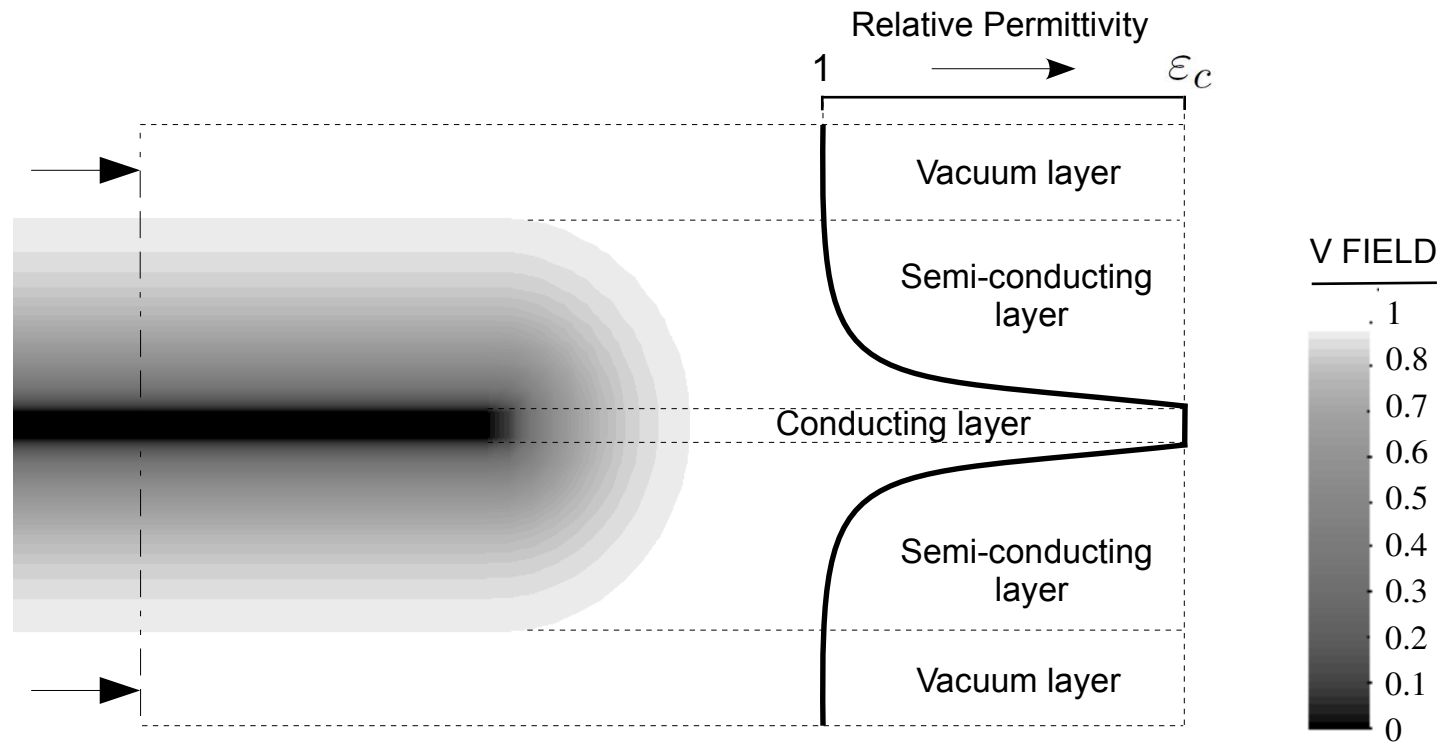

Figure 1: Schematic of a diffuse conducting layer. 


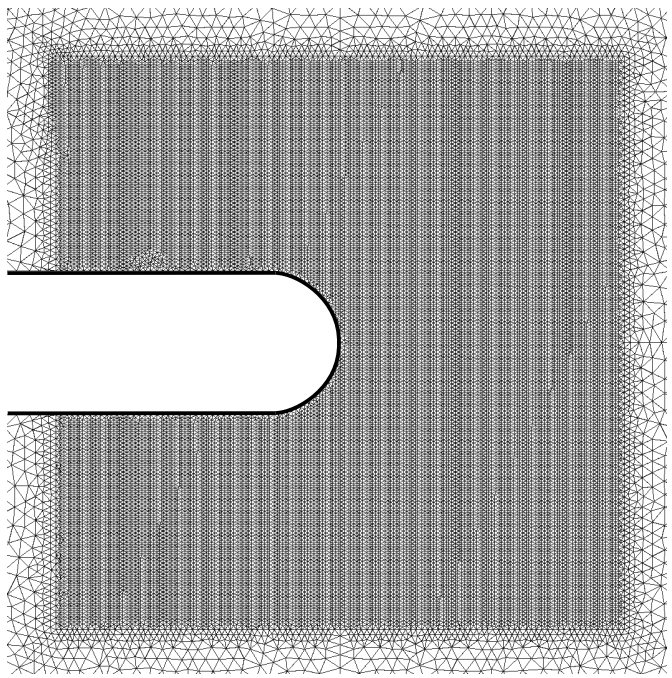

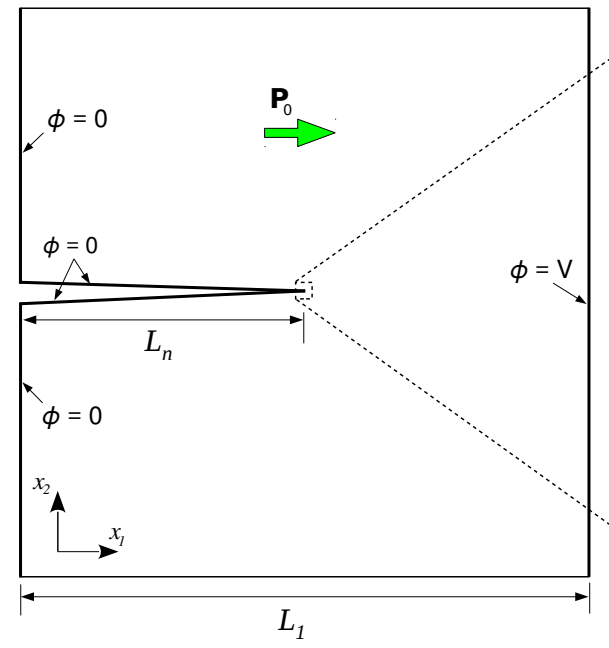

(a)

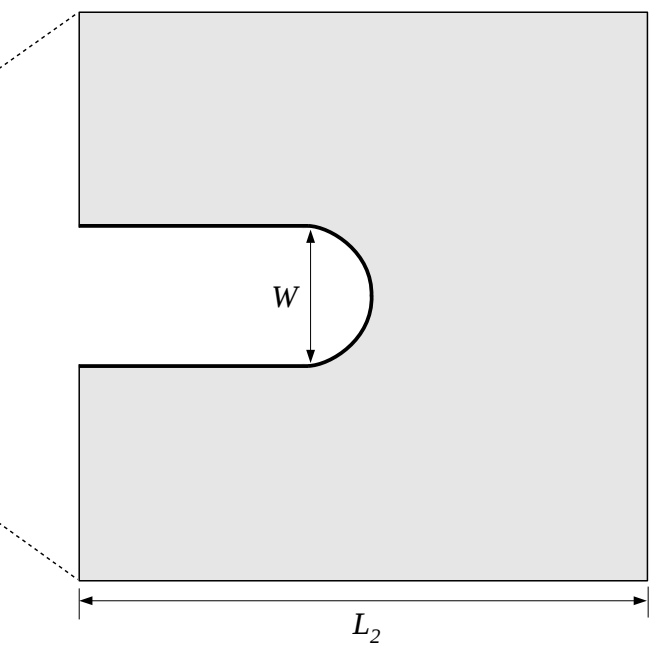

(b)

Figure 2: (a) Schematic of the computational domain and loading conditions. A pre-notch with the length of $L_{n}$ and the tip diameter of $W$ is considered in the model to facilitate the crack initiation. The initial polarization $\boldsymbol{p}_{0}$ is along the positive horizontal direction (b) small square area around the notch tip, where fracture is expected and (top) a finer mesh is generated in this area. Note that a positive applied electric field is oriented in the direction of the initial polarization 
(a)

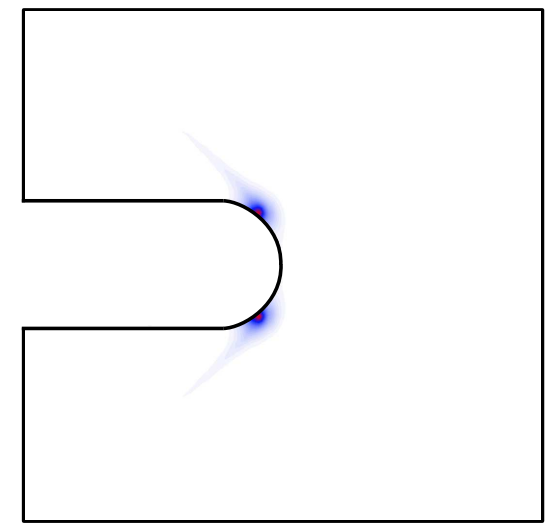

(b)

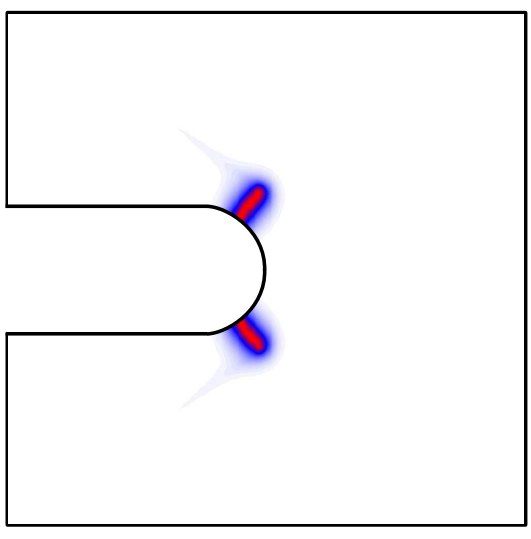

(c)

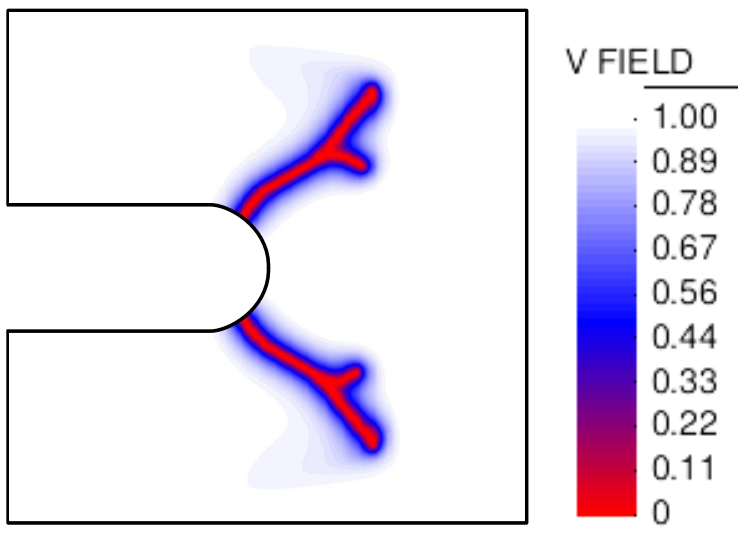

Figure 3: Three snapshots of the fracture evolution: contour plots of the field $v$ under positive electric fields (a) $E=1 \mathrm{KV} / \mathrm{mm}$ (b) $E=1.12 \mathrm{KV} / \mathrm{mm}$ (c) $E=1.4 \mathrm{KV} / \mathrm{mm}$. 
(a)

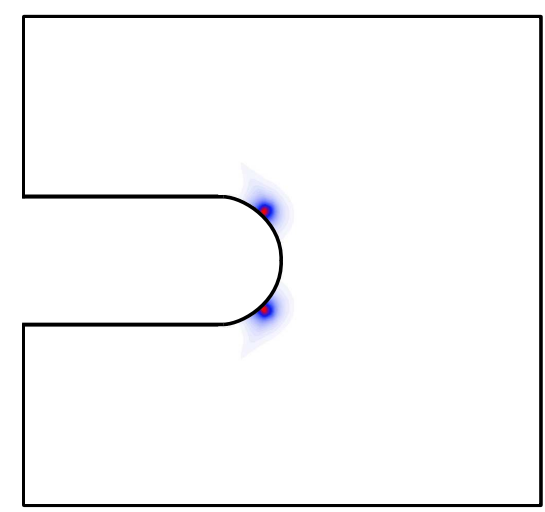

(b)

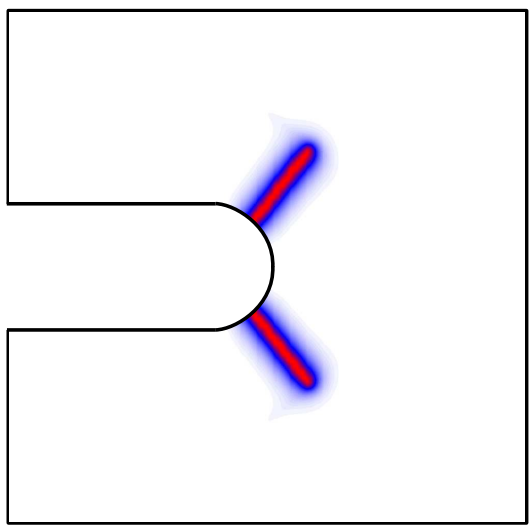

(c)
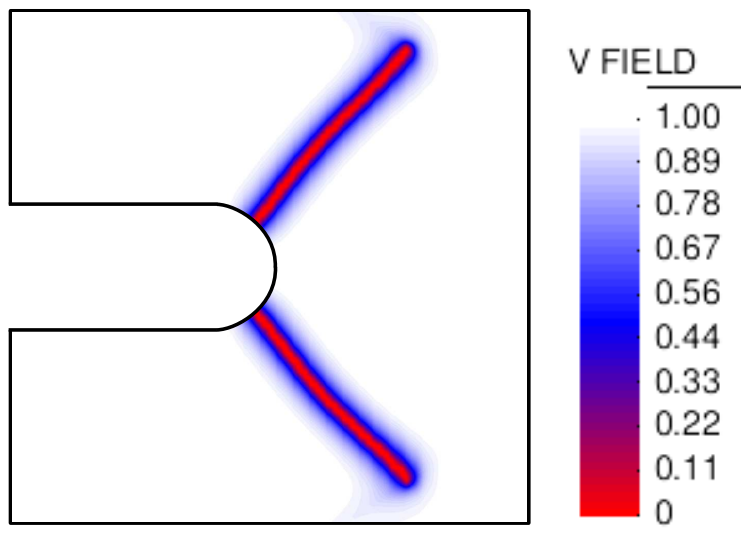

Figure 4: Three snapshots of the fracture evolution: contour plots of the field $v$ under negative electric fields (a) $E=-0.8 \mathrm{KV} / \mathrm{mm}$ (b) $E=-1.12 \mathrm{KV} / \mathrm{mm}$ (c) $E=-1.4 \mathrm{KV} / \mathrm{mm}$. 
(a)

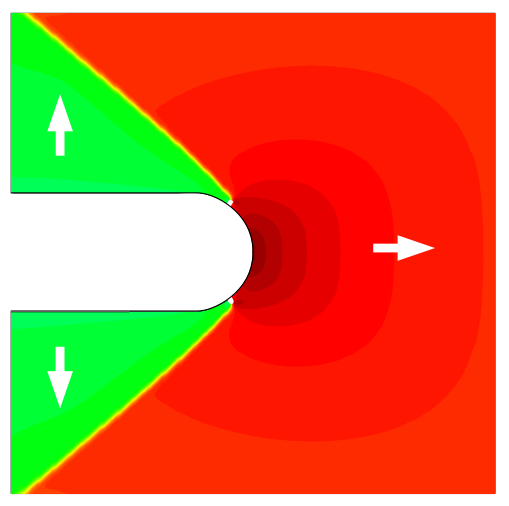

(b)

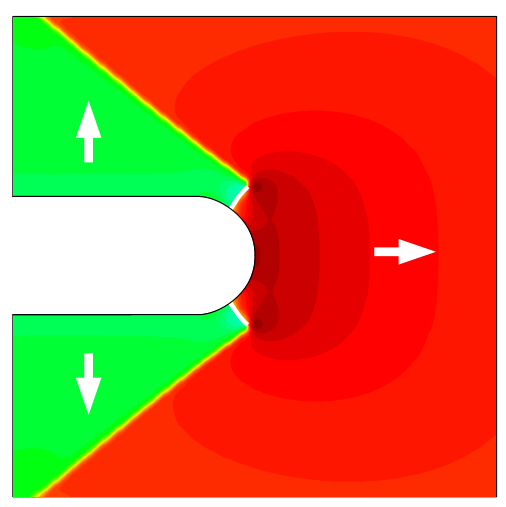

(c)

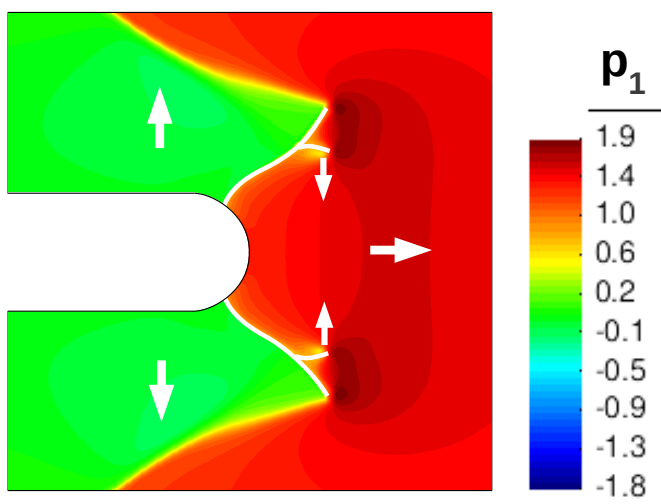

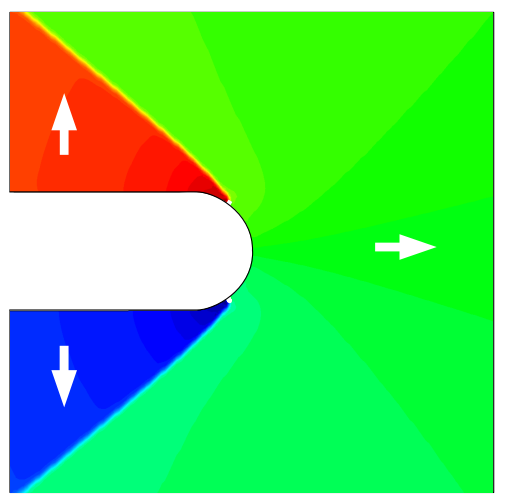
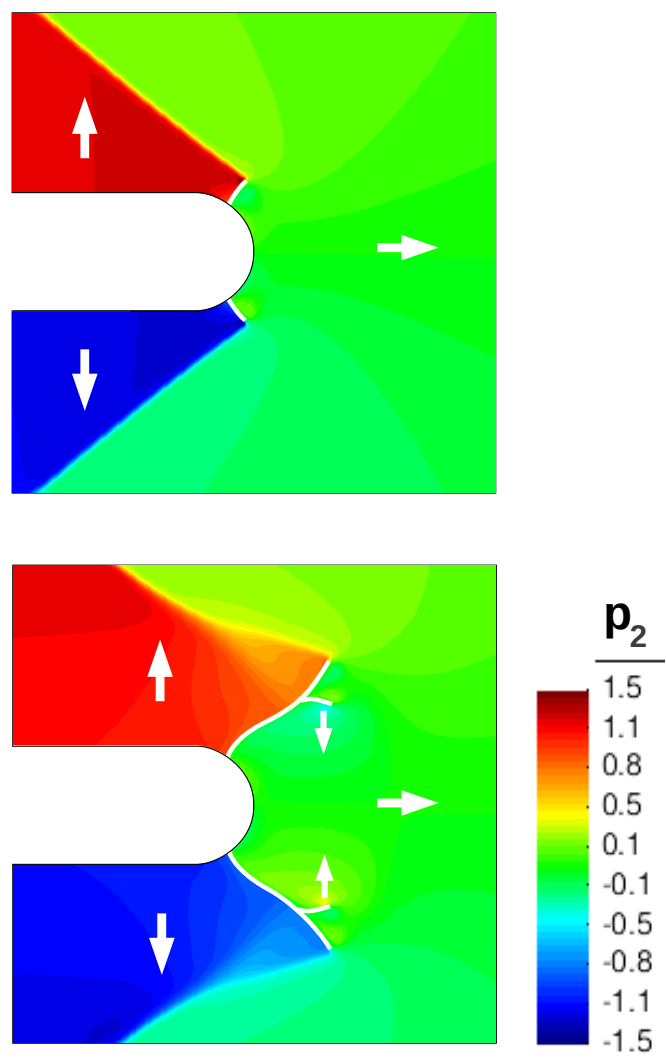

Figure 5: Three snapshots of the microstructure evolution in an area near the notch tip under positive electric fields (a) $E=1 \mathrm{KV} / \mathrm{mm}$ (b) $E=1.12 \mathrm{KV} / \mathrm{mm}$ (c) $E=1.4 \mathrm{KV} / \mathrm{mm}$. The left and right columns show the horizontal and vertical components of the normalized polarization, respectively. The white lines indicate the position of the cracks $(v=0)$. The domain orientations are indicated with white arrows. 
(a)

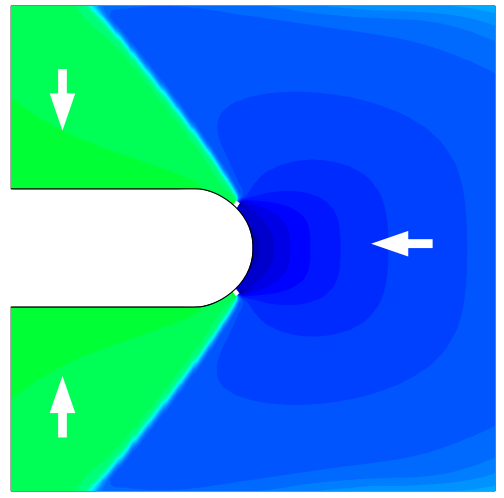

(b)

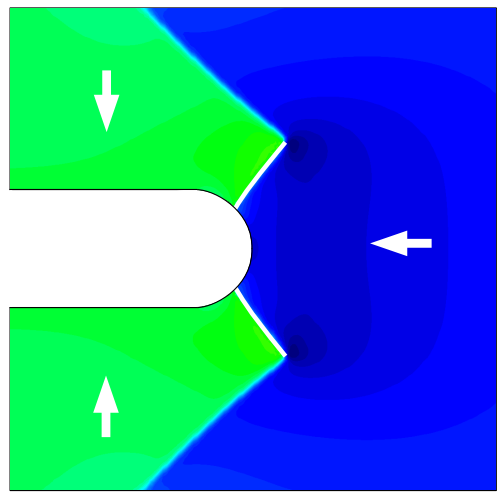

(c)

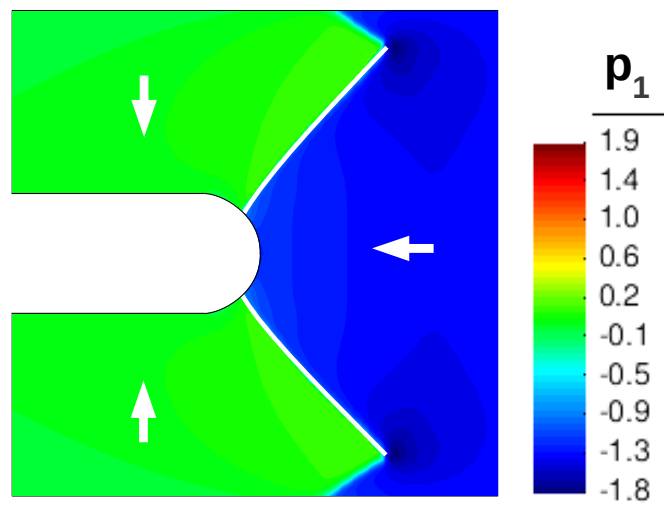

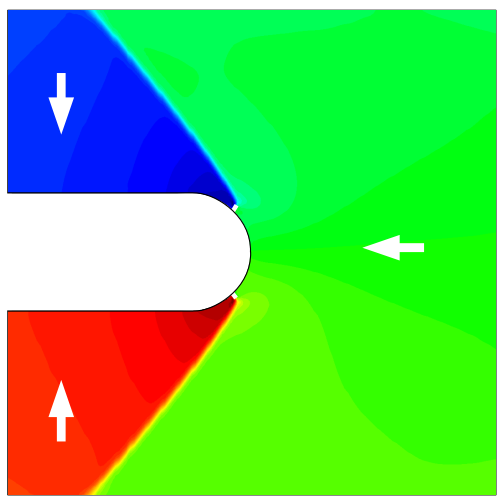
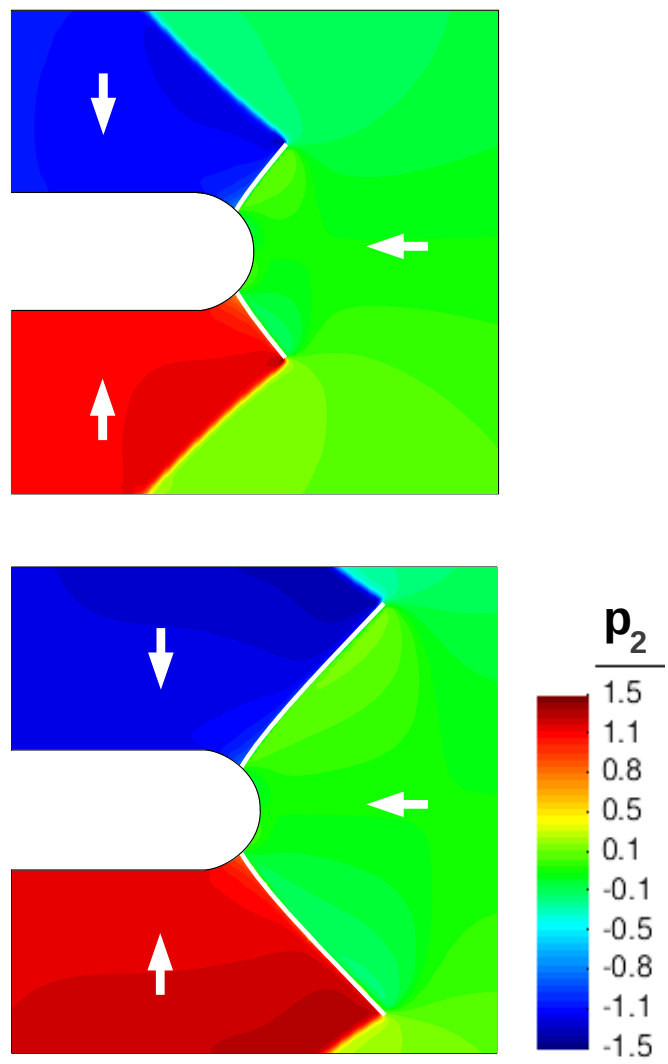

Figure 6: Three snapshots of the microstructure evolution in an area near the notch tip under negative electric fields (a) $E=-0.8 \mathrm{KV} / \mathrm{mm}$ (b) $E=-1.12 \mathrm{KV} / \mathrm{mm}$ (c) $E=-1.4$ $\mathrm{KV} / \mathrm{mm}$. The left and right columns show the horizontal and vertical components of the normalized polarization, respectively. The white lines indicate the position of the cracks $(v=0)$. The domain orientations are indicated with white arrows. 


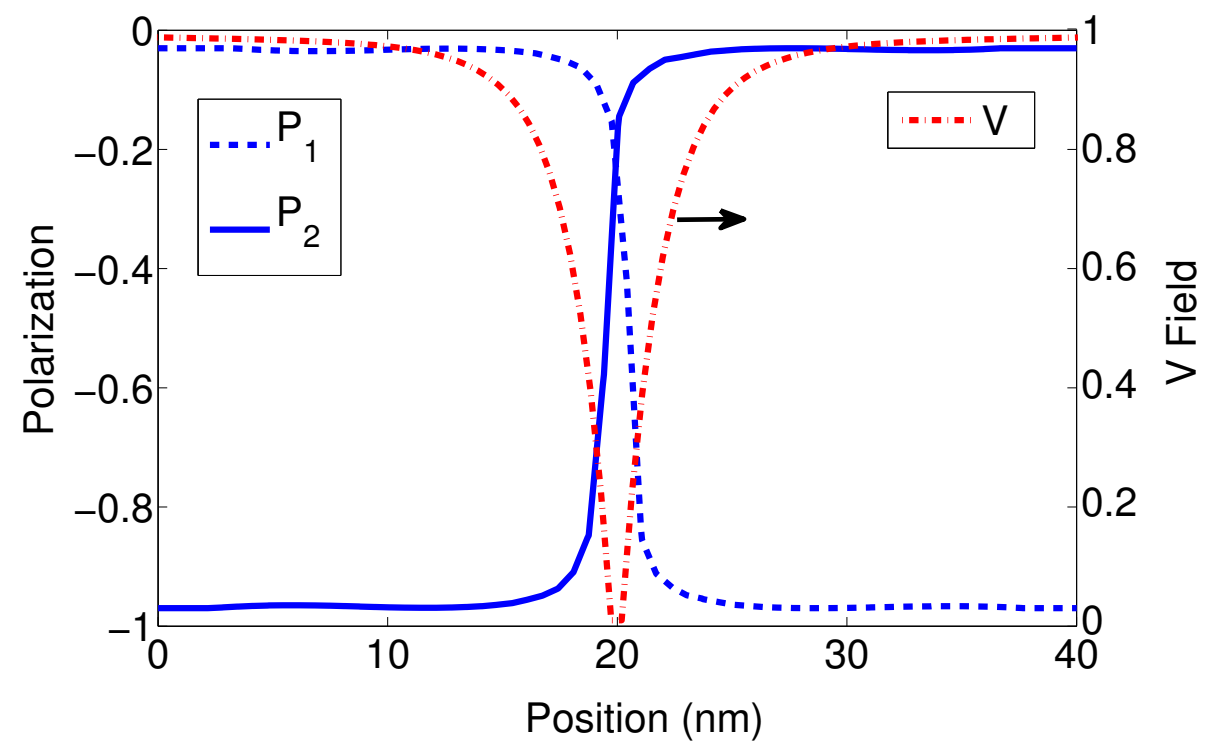

Figure 7: (Left) Vertical $\left(\mathrm{p}_{2}\right)$ and horizontal $\left(\mathrm{p}_{1}\right)$ components of the normalized polarization along a cross-section of the crack under the negative electric field. (Right) Field $v$ along the same section representing the smeared crack. The same sign of the polarization components on the two sides of the crack indicate the charge accumulation. 


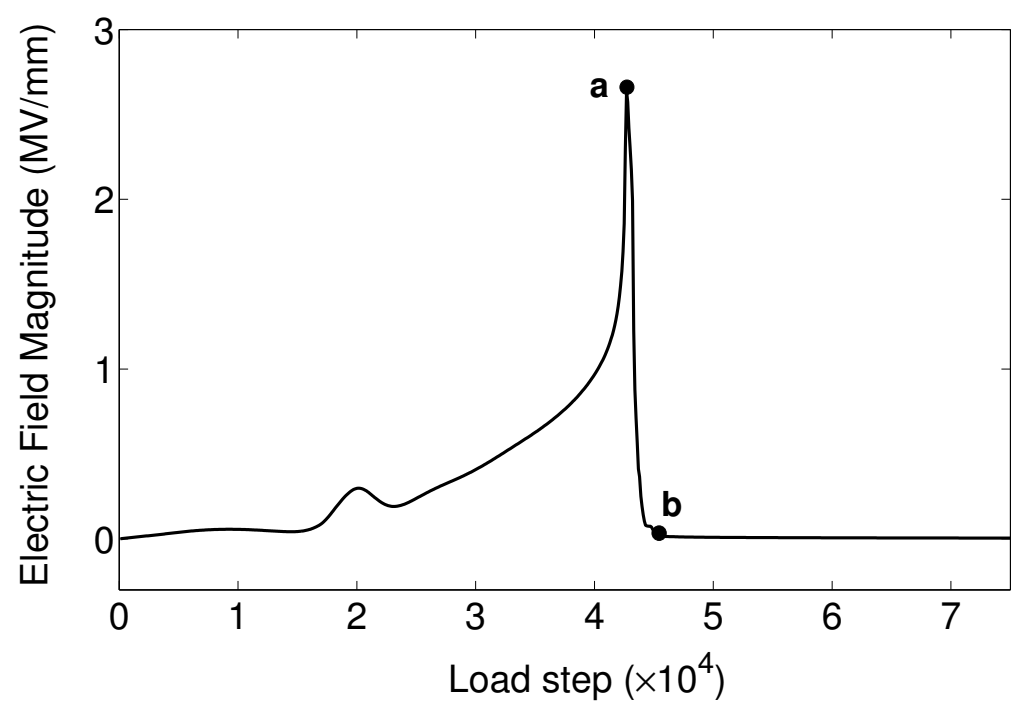

Figure 8: Electric field magnitude $|\mathbf{E}|$ as a function of the load step obtained at a point in the path of the conducting crack under the negative electric field. The crack reaches this point at load step a and passes through it at load step $\mathbf{b}$. 
Table 1: Normalized parameters

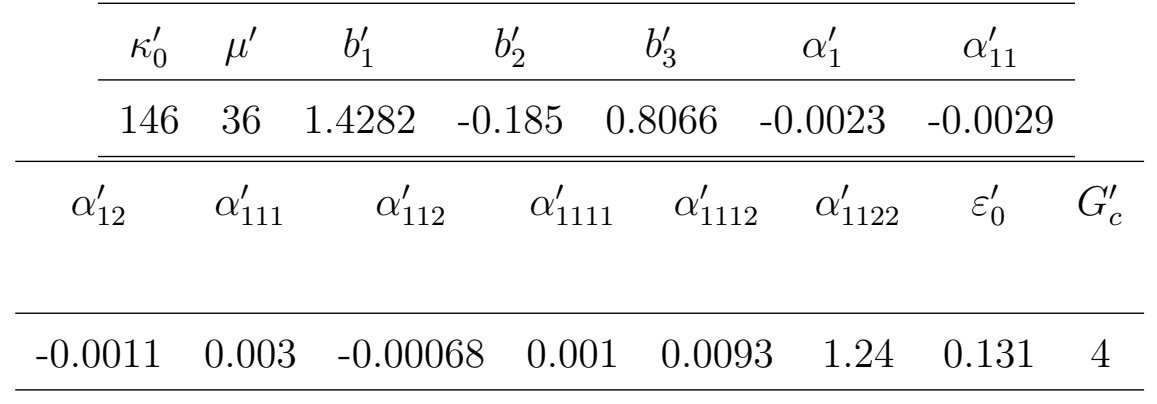




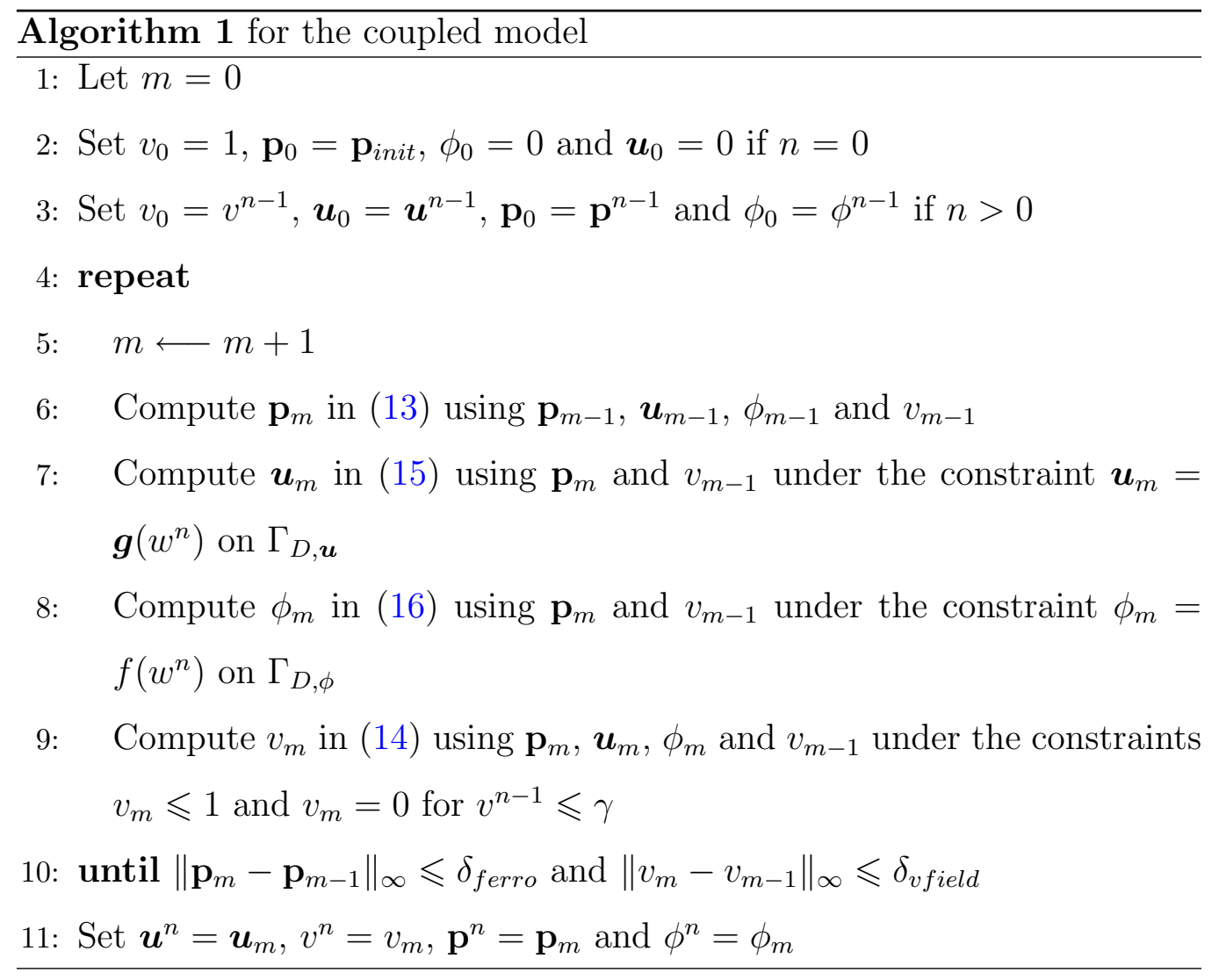

